\title{
Diagrams, Tensors and Geometric Reasoning
}

\author{
Jürgen Richter-Gebert • Peter Lebmeir
}

\section{Dedicated to the memory of Victor Klee, and in particular to his striving for conceptual simplicity}

Received: 30 June 2008 / Revised: 1 March 2009 / Accepted: 8 April 2009 /

Published online: 16 May 2009

(C) Springer Science+Business Media, LLC 2009

\begin{abstract}
Geometry and in particular projective geometry (and its corresponding invariant theory) deals a lot with structural properties of geometric objects and their interrelations. This papers describes how concepts of tensor calculus can be used to express geometric invariants and how, in particular, diagrammatic notation can be used to deal with invariants in a highly intuitive way. In particular we explain how geometries like euclidean or spherical geometry can be dealt with in this framework.
\end{abstract}

Keywords Projective geometry · Incidence theorems · Invariant theory · Tensors · Diagrams

\section{Introduction}

It is a very seldom experience that reading a mathematical article changes the way in which one thinks about a subject that one considered as "personally well understood." It so happened to us when we discovered a series of articles written by Jim Blinn that explained how to treat projective geometry on a diagrammatic level, in which the objects under consideration (points, lines, conics, algebraic curves, ...) are treated as vertices in a graph and their algebraic interrelations are represented by directed edges and some special types of nodes (see, for example, [2-14]). The vertices of the graphs in Blinn's papers are shorthands for tensors, and the edges represent contraction with

\footnotetext{
J. Richter-Gebert $(\bowtie) \cdot$ P. Lebmeir

Department of Mathematics, Technical University of Munich, Boltzmannstr. 3, 85748 Garching, Germany

e-mail: richter@ma.tum.de

url: http://www-m10.ma.tum.de/twiki/bin/view/Lehrstuhl/RichterGebert

P. Lebmeir

e-mail: lebmeir@ma.tum.de

url: http://www-m10.ma.tum.de/twiki/bin/view/Lehrstuhl/PeterLebmeir
} 
respect to an index in a tensor product. Each tensor represents a geometric object. Finally, every closed diagram (all ingoing and outgoing edges are actually connected to another vertex) turns out to be a number which is a projective invariant of a configuration of objects. In fact, interpreting the First Fundamental Theorem of Invariant Theory (see $[18,28,35]$ ) in this context, it turns out that every invariant can be expressed as a suitable function of closed diagrams. Tensorial approaches to projective geometry are well known as general and powerful tools (see [18]). Unfortunately, writing down concrete invariants in terms of tensors turns out to be a very clumsy business since most part of the information is hidden in upper and lower indices, indices of these indices, and indices of indices of indices, which makes it naturally very difficult to parse for a human reader. (Most probably, this is the reason why tensor approaches are rarely used in large parts of present day literature on geometry.) Tensor diagrams overcome these notational difficulties and help to unveil the underlying structure (and transformation rules) of the formulas involved. It is the main purpose of this article to give a brief introduction into this calculus.

We do not claim to prove any new results in this paper, nor do we claim to introduce any method that has not been presented elsewhere. Tensor calculus is very well known as a tool in projective geometry for more than hundred years; tensordiagrams are already used in the literature for quite a while. First attempts can be traced back even to Clifford and Sylvester in a letter exchange in connection with hyperdeterminants (for early sources, see [15, 19, 30], for more modern approaches, see, for instance, [17, 24, 27]). Also euclidean interpretations of tensor invariants are known since around the 1920s [31-34]. However, we were surprised of the power of diagram techniques even in very small examples, when all these concepts were put together and when we started to play around with them. So in essence the purpose of this article is to rebottle old wine and to demonstrate how small diagrams can be used to express interesting relations. We hope that the reader will enjoy these examples as much as we did, when we first started to explore their expressive power. ${ }^{1}$

Still there are some very serious reasons for doing research on this special way to represent invariants by diagrams. One ongoing research project that is pushed forward by the authors is the automatic recognition and classification of algebraic curves from high-precision sample data. Such problems arise, for instance, in the context of dynamic geometry software, where a geometric locus of a point under the movement of another point is calculated (see $[20,26]$ ). Such loci usually turn out to be algebraic curves and the geometric construction that generated them is often treated as a black box. One desire in this context is to automatically derive a name of the curve (like conchoid, lemniscate of Watt curve). After extracting the parameters of the curve from the sample data (a problem that has been dealt with in [21]), one is interested in extracting euclidean or projective invariants of the curve in order to compare these invariants to entries in a data base that relate names of curves to their invariants. Tensor diagrams turn out to be a very useful in this context to generate many invariants automatically without specific preknowledge on the structure of the curve [22, 23].

\footnotetext{
${ }^{1}$ It is a remarkable fact that tensor diagram techniques had a first and short heyday in the late 19th century among the people that dealt with classical invariant theory. Nowadays, they are most appreciated by physicists, where they are powerful tools in quantum field theory and turn out to be closely related to Feynman diagrams (compare, for instance, $[16,17,27])$.
} 
There are many other examples in which tensor diagrams are useful tools to study geometric properties of tensors. In Sect. 5.2 we will demonstrate very briefly how tensor diagrams are useful in the context of entanglement classification in quantum information theory. By this one gets a close relation of this field to projective geometry.

\section{Tensor Diagrams in a Nutshell}

It is not the aim of this paper to give a full introduction to the tensor diagram method. For this, we refer to the article series of Jim Blinn, which all together is about 200 pages. As first entry points, we recommend [2, 3]. Here we will just review the basic concepts for a reader who is already familiar with the basic concepts of projective geometry, homogeneous coordinates, and their transformations. We restrict ourselves to the case of real two-dimensional projective geometry $\left(\mathbb{R P}^{2}\right)$. The objects we consider will be points, lines, conics, and curves of higher algebraic degree. In most situations all our statements remain true for an arbitrary field of characteristic 0 , however the geometric pictures drawn will always refer to real situations. An important feature of the tensorial representation is that it is essentially coordinate-free in the sense that one can define a specific type of geometry by singling out specific objects (tensors) that should remain invariant with respect to its transformation group. By this essentially all geometric properties could be expressed by relations to these specific objects without ever referring to their concrete coordinates. The transformation rules govern the interrelations of the objects.

\subsection{Objects and Transformations}

We start with the situation in the projective plane. In projective geometry points are usually represented by homogeneous coordinates $p=\left(p_{1}, p_{2}, p_{3}\right)^{\mathrm{T}}$, where nonzero scalar multiples of a vector are considered to be equivalent. Likewise, lines are considered as homogeneous vectors $l=\left(l_{1}, l_{2}, l_{3}\right)^{\mathrm{T}}$. A point is incident to a line if the scalar product $\langle p, l\rangle=\sum_{i=1}^{3} p_{i} l_{i}$ vanishes. By this it is well known that, for instance, the join of two points $p, q$ can be represented by the cross product $p \times q$. Dually, the meet of two lines $l, m$ is $l \times m$. Three points $p, q, r$ are collinear if $\operatorname{det}(p, q, r)=0$, and a corresponding dual statement holds for three concurrent lines. The natural transformations in projective geometry are collineations for points (continuous transformations that preserve the collinearity of points). It can be shown that these transformations can always be expressed by a multiplication by a $3 \times 3$ matrix $T$. Again, matrices differing only by a nonzero scalar multiple represent identical transformations. Since incidences of points and lines should be preserved under projective transformations, this implies the transformation behavior of vectors representing lines as well. If a projective transformation on the set of points is expressed by $p \mapsto T p=$ : $\tau(p)$, then the corresponding operation on the lines must be $l \mapsto\left(T^{-1}\right)^{\mathrm{T}} l=: \tau(l)$. Then and only then we have $\langle\tau(p), \tau(l)\rangle=\left\langle T p,\left(T^{-1}\right)^{\mathrm{T}} l\right\rangle=p^{\mathrm{T}} T^{\mathrm{T}}\left(T^{-1}\right)^{\mathrm{T}} l=\langle p, l\rangle$, and transformations preserve incidences.

Let us step back for a moment and look at the structure of the expressions involved. We have (so far) three types of objects: points, lines, and transformations. Points and 
lines are a one-dimensional array of numbers (a list of coefficients) and a transformation is a two-dimensional array of numbers. Transformations of points and lines have to be treated differently. Let us consider the points, lines, and transformations as arrays and just represent them by $\left(p_{i}\right)_{i=1 \ldots 3},\left(l_{i}\right)_{i=1 \ldots 3}$, and $\left(T_{i j}\right)_{i=1 \ldots 3 ; j=1 \ldots 3}$. For the moment, we furthermore set $S=T^{-1}$ with the components $\left(S_{i j}\right)_{i=1 \ldots 3 ; j=1 \ldots 3}$. With this our above expression $\left\langle T p,\left(T^{-1}\right)^{\mathrm{T}} l\right\rangle$ may then be expanded as (check it)

$$
\sum_{i=1}^{3} \sum_{j=1}^{3} \sum_{k=1}^{3} T_{j i} p_{i} S_{k j} l_{k} .
$$

Observe that $p_{i}$ shares the second index with $T_{j i}$, and $l_{k}$ shares the first index with $S_{k j}$. The latter property corresponds to the fact that $l$ is transformed by the transposed of $T^{-1}=S$. For more complicated expressions, we get even more summations and more indices. Multidimensional arrays of numbers are essentially mathematical tensors. In this way we associated each object with a certain tensor in the above expression. There is a nice notational trick that helps us to get rid of the many summation signs. By the Einstein-sum-convention we can split the indices into suband superscripts and sum over each index letter that occurs as well as sub- and as superscript. Thus we could write $\langle p, l\rangle=p_{i} l^{i}$ or, for our last example,

$$
\left\langle T p,\left(T^{-1}\right)^{\mathrm{T}} l\right\rangle=\left\langle T p,(S)^{\mathrm{T}} l\right\rangle=p_{i} T_{j}^{i} S_{k}^{j} l^{k} .
$$

Rowness, columnness, and matrix transpositions and even the multiplication order is now completely encoded into the positions and labels of the indices. This is an instance of the classical way of dealing with projective geometric objects as tensors.

There is a good reason why this kind of notation only rarely shows up in geometry literature: It is hard to read. Most of the information is transferred to the exact positions of the indices. Tensor notation is even commutative: $p_{i} T_{j}^{i} S_{k}^{j} l^{k}=S_{k}^{j} T_{j}^{i} l^{k} p_{i}$. Also the order (in classical sense) of multiplications is encoded by the indices. The situation becomes worse if one has to consider indexed sets of indices (which happens quite often) or powers of tensors (where it becomes hard to distinguish superscripts from powers). Finally there are only 26 letters in our alphabet, so it may very often happen that one simply runs out of letters for indices, especially, when some are blocked for other purposes.

This is the point where diagram notation enters as a useful tool. An index $i$ in the above notation carries essentially the information which tensor is multiplied by which. The super- and sub-script furthermore indicate if the object has to be transformed by left-multiplication with $T$ or with $\left(T^{-1}\right)^{\mathrm{T}}$. Let us represent each tensor by a node in a graph. Connect two tensors by an arrow if they share an index. Here the arrow will always go from the sub-script position to the super-script position. Indices that occur only once in a formula will be denoted by a free arrow that has a loose end. We may label the arrows by the corresponding indices, however, this is not even necessary since the main purpose of the indices was to designate which tensor shares a summation index with which other and this information is already present in the position of the arrows. Memorizing $S=T^{-1}$, our running example may be 
encoded as

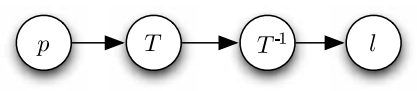

A point corresponds to a node with an outgoing arrow. A transformation has an incoming and an outgoing arrow. A line is a node with an incoming arrow. We call all outgoing arrows covariant and incoming arrows contravariant. What scalar products and matrix multiplications had been in the classical notation now becomes simply a matter of connecting the arrows. The point $p$ being transformed by the matrix $T$ is denoted by $P \rightarrow T \rightarrow$. The line $l$ being transformed by $\left(T^{-1}\right)^{\mathrm{T}}$ results in (l) $\leftarrow$. Performing the scalar product between these two expressions is simply soldering the two free arrows.

The inner part of the entire diagram corresponds to the matrix multiplication $T \cdot T^{-1}=E$. Thus we can replace

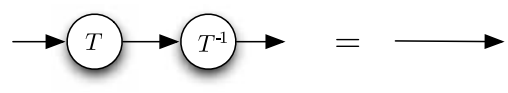

and get the identity

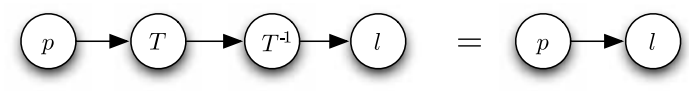

This expresses just the fact that the scalar product $\rightarrow \rightarrow$ remains invariant under projective transformation of the objects.

\subsection{The $\delta$-Tensor}

There is one ingredient in the classical theory of tensors that remains almost unnoticed in the context of tensor diagrams: The so-called $\delta$-tensor $\delta_{i}^{j}$. This is a tensor that is defined as the Kronecker-Delta. Thus it is a two-dimensional number array defined by the property $\delta_{i}^{i}=1$ and $\delta_{i}^{j}=0$ for $i \neq j$. Thus this tensor plays the role of a unit matrix. In the tensor world it "just" relabels the indices. For instance, we have $p_{j} \delta_{i}^{j}=p_{i}$ and $p_{j} \delta_{i}^{j} l^{i}=p_{i} l^{i}$. So the $\delta$-tensor can be used to connect a covariant and a contravariant tensor with different indices. In the context of tensor diagrams a $\delta$-tensor plays the role of an intermediate extra arrow and remains essentially unnoticed. We will see that $\delta$-tensors still play an essential role in this context when we start to transform diagrams into different looking equivalent ones.

\section{$2.3 \varepsilon$-Tensors}

So far the expressive power of our diagrams is not very rich. We can only express linear chains of objects with transformations in-between. The situation changes drastically if we introduce the so called $\varepsilon$-tensors. The size and number of connecting 
arrows of an $\varepsilon$-tensor depends on the dimension of the ambient space. For the projective plane, an $\varepsilon$-tensor is a $3 \times 3 \times 3$ array with either all arrows covariant or all arrows contravariant. The covariant $\varepsilon$-tensor is defined by the facts that we have $\varepsilon_{123}=1$ and that it is completely antisymmetric (i.e., interchanging two indices flips the sign of the entry). By this we get

$$
\varepsilon_{123}=\varepsilon_{231}=\varepsilon_{312}=-\varepsilon_{132}=-\varepsilon_{321}=-\varepsilon_{213}=1 \text {. }
$$

All remaining entries must be zero (as a consequence of antisymmetry). The contravariant $\varepsilon$-tensor is defined analogously. We represent $\varepsilon$-tensors by the following two diagram nodes:

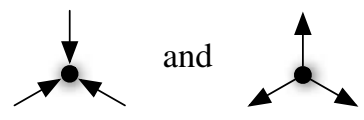

It is not too difficult to check that we get the following interpretation for diagrams. If $l=p \times q$ is the join of two points and if $p=l \times m$ is the meet of two lines, we get

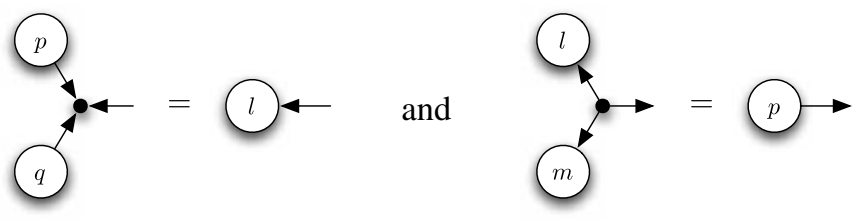

The first diagram represents an object with an incoming arrow. Thus it is contravariant, "line-like" so to say. The second diagram represents an object with an outgoing arrow. Thus it is "point-like." A simple calculation shows that performing the necessary summations, we obtain exactly the vector products of the points. Another remarkable identity is

$$
p_{i} q_{j} r_{k} \varepsilon^{i j k}=\operatorname{det}\left(\begin{array}{ccc}
p_{1} & q_{1} & r_{1} \\
p_{2} & q_{2} & r_{2} \\
p_{3} & q_{3} & r_{2}
\end{array}\right)
$$

The $\varepsilon$-tensor simply selects all six summands that are present in the evaluation of a determinant. In diagram notation determinants of three points or three lines read

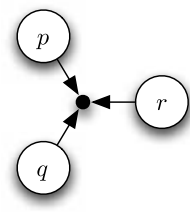

and

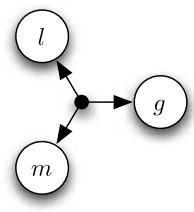

\subsection{How Do $\varepsilon$-Tensors Transform?}

$\varepsilon$-tensors have a remarkable transformation behavior. Applying a projective transformation to an $\varepsilon$-tensor simply results in a tensor that is a multiple of the $\varepsilon$-tensor. The 
scaling factor is the determinant of the transformation matrix. Thus we have

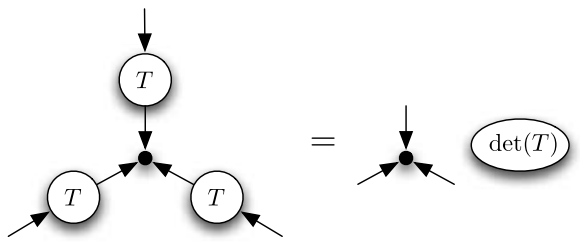

Here the oval marked $\operatorname{det}(T)$ on the right of the equation means that the $\varepsilon$-tensor is multiplied by this scalar, a tensor with no in- and no outgoing arrows. There are several ways to convince oneself of this formula. One way is simply to perform all the summations and expand the resulting terms. Another way is to consider the transformation behavior of a configuration with three points plugged into the three entries of the $\varepsilon$-tensor. For a detailed discussion, see $[2,3]$.

Thus under a projective transformation in essence an $\varepsilon$-tensor reproduces itself. If we also consider $\varepsilon$-tensors identified with scalar multiples of themselves, the $\varepsilon$ tensor is a remarkable object with the property of being invariant under arbitrary projective transformations. In a way the role of the $\varepsilon$-tensor in projective geometry is comparable to the role of the line at infinity in affine geometry. It stays invariant under all transformations of the group. We will later on see that this analogy carries even further.

The behavior of the $\varepsilon$-tensor allows us to form complicated diagrams that encode projectively invariant properties. If we use concrete tensors (geometric objects) in a closed diagram (no free arrows), it will always evaluate to a specific number. The actual absolute value of this evaluation does not tell a lot, since it may be scaled by replacing a tensor by one of its multiples. However, if such a diagram turns out to be zero, it encodes a projective property among the objects involved. For instance, the vanishing of the diagram $\rightarrow(l)$ represents the fact that $p$ and $l$ are coincident. There are circumstances under which also the sign of the number carries some information. This may happen if each tensor occurs an even number of times.

The $\varepsilon$-tensor gives us the possibility to generate branched diagrams and diagrams that even contain loops. The following theorem (cf. [18]) tells us that such closed diagrams always correspond to projectively invariant properties.

Theorem 1 Let $\mathcal{D}$ be a closed tensor diagram consisting of tensors that represent geometric objects and $\varepsilon$-tensors. If the projective space is transformed by $\tau$ with corresponding matrix $T$, then the evaluation of the diagram is scaled by a factor $\operatorname{det}(T)^{k}$, where $k$ is the difference of covariant and contravariant $\varepsilon$-tensors.

Proof Rather than giving a strictly formal proof, we confine ourselves here with a "proof by example" that demonstrates the main principles in diagram notation. We consider the following diagram that consists of four points, one line, and three 
$\varepsilon$-tensors (two of them are contravariant, and the other is covariant):

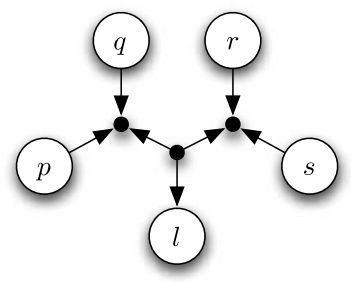

How does the evaluation change if we transform the geometric objects by a projective transformation $\tau$ given by the pair of matrices $\left(T, T^{-1}\right)$ ? Covariant arrows (our points) of geometric objects have to be transformed by $T$, contravariant arrows (or lines) have to be transformed by $T^{-1}$, and we obtain

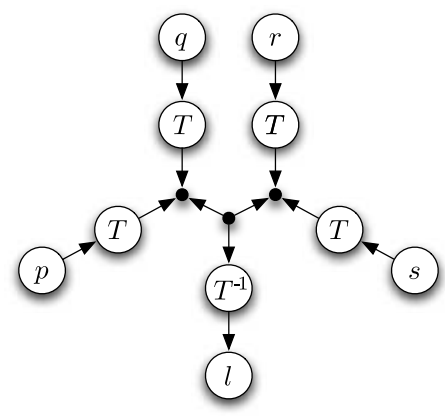

For evaluating this diagram, we "blow up" the interior arrows by replacing them by a pair of transformations $\rightarrow \mathrm{T}^{-1} \rightarrow T \rightarrow$. After this procedure our diagram looks as follows:

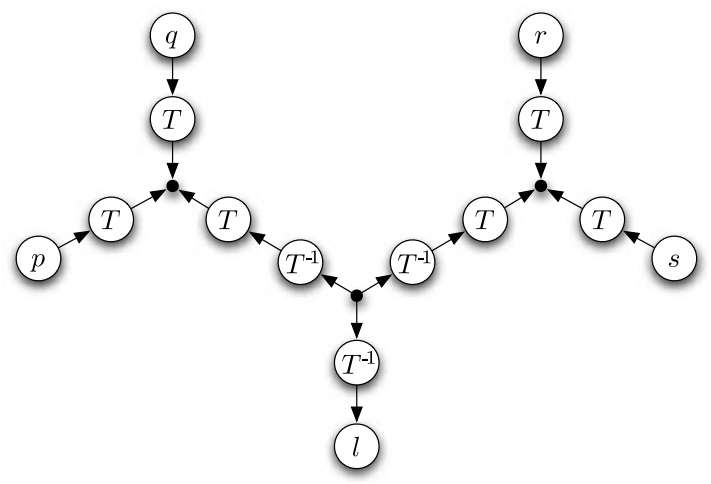

Now each contravariant $\varepsilon$-tensor is surrounded by a halo of $T$-tensors, and each covariant $\varepsilon$-tensor is surrounded by $T^{-1}$-tensors. We can replace each such $\varepsilon$-tensor together with its surrounding transforming matrices by an $\varepsilon$-tensor multiplied by the 
corresponding determinant. By this we get

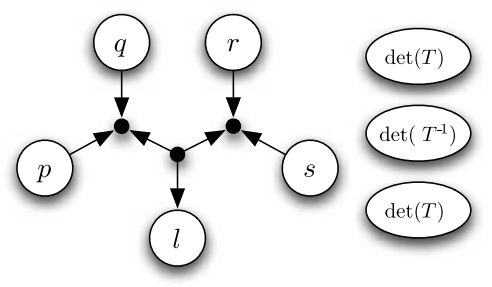

This is just our original diagram multiplied by $\operatorname{det}(T)^{2} \cdot \operatorname{det}\left(T^{-1}\right)$. Since the determinant of $T^{-1}$ is the inverse of the determinant of $T$, the final factor is $\operatorname{det}(T)$. In general we obtain a factor that is $\operatorname{det}(T)^{k}$, where $k$ is the difference of the number of contravariant minus the number of covariant $\varepsilon$-tensors.

\subsection{Invariants}

Every closed diagram represents a projectively invariant property. This means that if we have tensors $a, b, c, \ldots$ that make this diagram evaluate to zero, then the diagram remains zero if the tensors are replaced by simultaneous projective transforms $\tau(a), \tau(b), \tau(c), \ldots$ In essence the converse is also true. Polynomial functions that are (relative) invariants of a given set of geometric objects will always be expressible as a linear combination of closed diagrams. This is a consequence of a version of the First Fundamental Theorem of Invariant Theory on the level of tensors (as stated and proved, for instance, in [18]). Due to the fact that our current setup is on the level of homogeneous coordinates and of projective transformations, where we identify nonzero scalar multiples, certain technical difficulties in stating the term of an invariant arise. We avoid these difficulties by referring to vector configurations (where we do not identify multiples of the objects) in contrast to homogeneous configurations. Let $a_{1}, \ldots, a_{N}$ be the collection of coordinate entries of a collection of geometric objects. We represent the objects by suitable tensors. For a linear transformation $T$, we denote by $a_{1}^{\prime}, \ldots, a_{N}^{\prime}$ the corresponding transformed coordinate entries. The $a_{i}$ 's are derived by transforming the corresponding tensor by $T$ for covariant and by $T^{-1}$ for contravariant indices. A polynomial $\varphi\left(a_{1}, \ldots, a_{N}\right)$ is called a relative invariant for the set of regular linear transformations $T$ if we have $\varphi\left(a_{1}^{\prime}, \ldots, a_{N}^{\prime}\right)=\operatorname{det}(T)^{g} \varphi\left(a_{1}, \ldots, a_{N}\right)$ for all choices of $a_{1}, \ldots, a_{N}$ and $T$. The integer $g$ is called the weight of the invariant. If $g=0$, the invariant is called an absolute invariant. Now the First Fundamental Theorem states that each such invariant can be expressed as a linear combination of (multihomogeneous) closed diagrams (see $[18,25])$.

Properties that are invariant under projective transformations may be expressed as the vanishing of a polynomial invariant in the above sense (vanishing of invariants are not altered by taking scalar multiples). If one likes to measure projectively invariant functions (like, for instance, the cross ratio), one has to consider rational functions that are formed by dividing two polynomial invariants that involve exactly the same number of objects the same number of times. 


\subsection{Replacement Rules}

The representation of invariants is by far not unique. The same invariant may be represented by completely different (linear combinations of) diagrams. This effect is common to invariant theory. For instance, in the classical projective invariant theory of configurations of points there exist certain syzygies (for instance, GrassmannPlücker relations) that are always zero, independent of the value of the objects. Adding such a syzygy to an invariant does not change the meaning of the invariant but its concrete algebraic appearance. On the level of tensor diagrams one can nicely separate the structural components of a syzygy from its coordinate entries. For instance, the Grassmann-Plücker relation

$$
\operatorname{det}(a, b, c) d-\operatorname{det}(a, b, d) c+\operatorname{det}(a, c, d) b-\operatorname{det}(b, c, d) a=0
$$

translates to the tensor diagram equation
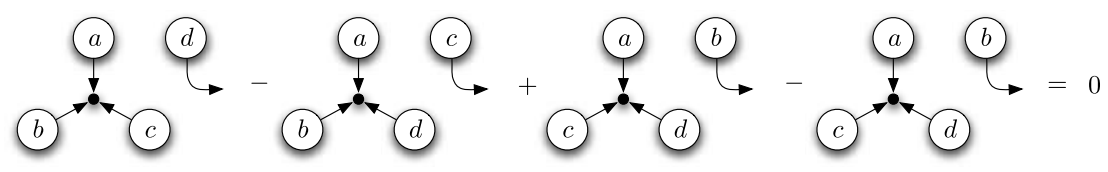

Here sub-diagrams in each summand have to be multiplied. The four letters $a, b, c, d$ occur in each single summand, and each single summand has exactly one outgoing arrow. As a matter of fact, one can neglect the specific points that are used in the summands as long as it is made sure that the ends of the $\varepsilon$-tensors and the free arrows are connected to consistent parts of a surrounding diagram. If we rewrite the above equation in the tensor language, we get

$$
a_{i} b_{j} c_{k} d_{l} \varepsilon^{i j k} \delta_{m}^{l}-a_{i} b_{j} c_{k} d_{l} \varepsilon^{i j l} \delta_{m}^{k}+a_{i} b_{j} c_{k} d_{l} \varepsilon^{i k l} \delta_{m}^{j}-a_{i} b_{j} c_{k} d_{l} \varepsilon^{j k l} \delta_{m}^{i}=0
$$

where $m$ plays the role of the outgoing index. We can factor out the points and derive

$$
\varepsilon^{i j k} \delta_{m}^{l}-\varepsilon^{i j l} \delta_{m}^{k}+\varepsilon^{i k l} \delta_{m}^{j}-\varepsilon^{j k l} \delta_{m}^{i}=0 .
$$

This is the essential structural part of the Grassmann-Plücker relation. It tells us that for any (!!) diagram that involves an $\varepsilon$-tensor and a disjoint arrow, we get an equivalent diagram by summing three copies of the original diagram, in which the $\varepsilon$-tensor and the arrow have been suitably rewired. One might wonder where there is a use of this replacement of a diagram by the sum of three diagrams with equivalent complexity. A first answer to this is that this mimics addition of the GrassmannPlücker relation or a similar syzygy to an invariant, which is a powerful operation in classical projective geometry and invariant theory (for instance, it is the basis of the straightening algorithm (see [28]). However there is also a second answer: The above equation is not the only one of a similar type-there are some of them that greatly help to cut down the complexity of diagrams. The following one is (for the projective plane) perhaps the most elementary and useful one. It is the so-called $\varepsilon$ - $\delta$-rule

$$
\varepsilon^{i j k} \varepsilon_{l m k}=\delta_{l}^{i} \delta_{m}^{j}-\delta_{m}^{i} \delta_{l}^{j} .
$$


(If you want, you can prove it by brute-force expansion of the terms.) Translated into diagram language this becomes
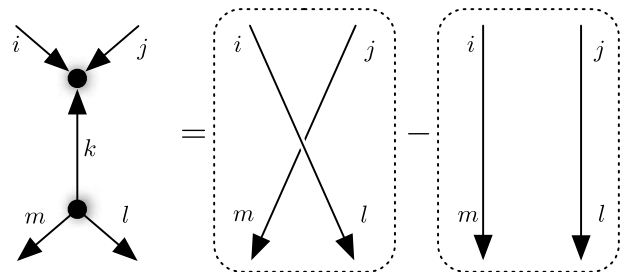

Thus the $\varepsilon$ - $\delta$-rule can take a diagram that contains two adjacent (co- and contravariant) $\varepsilon$-tensors and replace it by the difference of two diagrams in which these $\varepsilon$-tensors have vanished. We will see later on how useful this operation is. Perhaps one word of caution is required here. $\varepsilon$-tensors are antisymmetric tensors. This means that interchanging two indices corresponds to a multiplication by -1 . When dealing with diagrams, one has to be careful not to interchange two arrows of an $\varepsilon$-tensor without changing the sign of the corresponding diagram. As a little finger exercise, the reader is invited to prove the identity
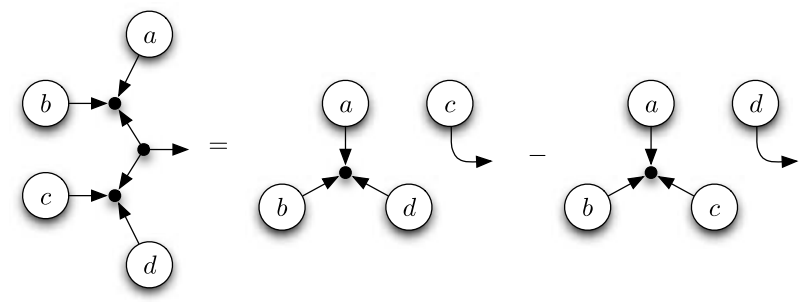

by using the $\varepsilon$ - $\delta$-rule. This equation corresponds to the fact that

$$
\operatorname{meet}(\operatorname{join}(a, b), \operatorname{join}(c, d))=\operatorname{det}(a, b, c) d-\operatorname{det}(a, b, d) c .
$$

It is even possible to derive the Grassmann-Plücker relations by suitable application of the $\varepsilon$ - $\delta$-rule (try it!).

\section{Projective Geometry}

This section is devoted to applying the calculus of tensor diagrams to projective geometry. Here we can just give a glimpse of the expressive power of tensor diagrams. For a wealth of interesting examples, we refer to the article series by Jim Blinn on this subject. Here we try to give a few interesting examples that are in essence disjoint from the example list covered by Blinn.

\subsection{The Simplest Invariant}

Let us start with an extremely simple closed diagram and an analysis of its geometric meaning. The simplest closed diagram containing a nonscalar tensor-node just 
contains one tensor and one arrow. Thus the tensor must have one ingoing and one outgoing edge and thus is a transformation tensor

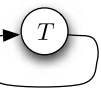

If this tensor diagram vanishes, the transformation must have a specific projective property. Algebraically this corresponds to evaluating $T_{i}^{i}=T_{1}^{1}+T_{2}^{2}+T_{3}^{3}$, which is just the trace of the matrix. In fact transformations with trace 0 have special projective properties.

We can observe a first special property of trace-zero transformations if we apply the $\varepsilon$ - $\delta$-rule to the pair of $\varepsilon$-tensors in following diagram:

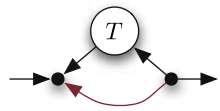

We obtain the following chain of equivalent diagrams:

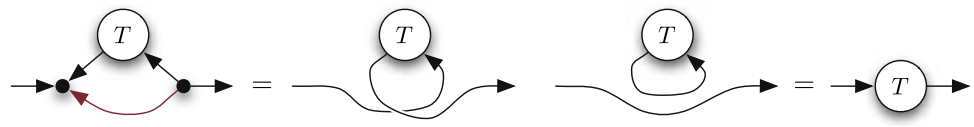

The first equation sign comes from the $\varepsilon$ - $\delta$-rule. In the next expression the second summand vanishes according to the trace-zero condition. Unwinding the diagram, we see that the right side is just equivalent to the original transformation. Thus, for a trace-zero transformation, we can exchange any occurrence of $T$ by the more complicated expression on the left. (One might wonder what is the use of this. We will see an application of this in the next theorem.)

Transformations with vanishing traces can also be characterized via incidence relations of images and pre-images of three points. This geometric property is stated in the following theorem.

Theorem 2 Let $\tau$ be a projective transformation in $\mathbb{R P}^{2}$ whose corresponding matrix $T$ satisfies trace $(T)=0$. Let $a$ and $b$ be two arbitrary points, and $a^{\prime}, b^{\prime}$ their images under $\tau$. Define $c$ as the meet of $\operatorname{join}\left(a, b^{\prime}\right)$ and join $\left(a^{\prime}, b\right)$. Then the image $c^{\prime}$ of $c$ lies on the join of $a$ and $b$.

Proof It is not too hard to prove this fact entirely by methods of linear algebra. However, we will investigate how the result can be obtained by shuffling around diagrams. The incidence relation stated in the theorem can be expressed as follows: For every trace-zero transformation $T$ and for arbitrary points $a$ and $b$, the following diagram 
vanishes:

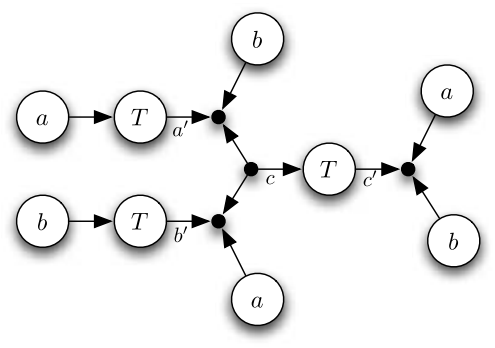

The small letter at the arrows refer to the corresponding point in the theorem $\left(a^{\prime}\right.$ is the image of $a ; b^{\prime}$ is the image of $b$; $c$ is the join of the two lines; and $c^{\prime}$ is the image of $c$ ). The diagram encodes the construction of the theorem from the left to the right. The rightmost $\varepsilon$-tensor expresses that $c^{\prime}$ is incident to the join of $a$ and $b$. So, how do we prove that this diagram always vanishes? Applying the $\varepsilon-\delta$-rule to any of its internal arrows decomposes the diagram into smaller units, and we have no chance to apply the trace-zero condition. However, we can proceed as follows: First we exchange one occurrence of $T$ by the more complicated diagram we mentioned above and obtain

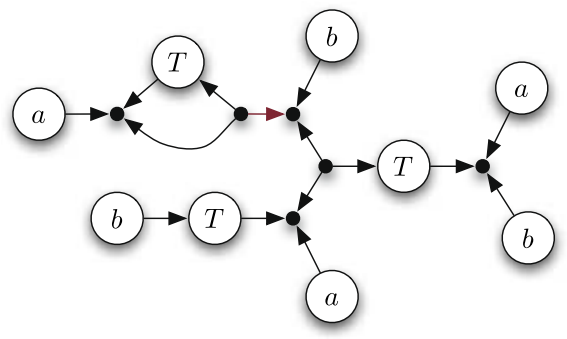

This diagram is equivalent to the above one if $T$ has vanishing trace. Applying the $\varepsilon$ - $\delta$-rule at the right place in this diagram gives

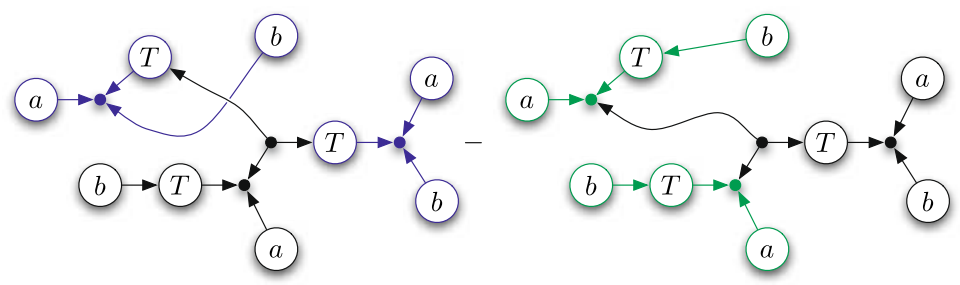

Now we see that obviously both summands vanish: In the first summand we have twice the subgraph of an $\varepsilon$-tensor connected to $a, b$, and $T$. Plugging these (up to a sign) identical sub-diagrams in the same $\varepsilon$-tensor must give us a zero. In the second summand it is the same with the two identical sub-structures of an $\varepsilon$-tensor with $a$ and $b$ connected to $T$. Thus the entire diagram must be identically equal to zero. 


\section{$3.2 \varepsilon$-Cycles}

As a second example, let us consider closed rings of $\varepsilon$-tensors (alternatingly co- and contravariant) and investigate their geometric meaning under various aspects. Since all our arrows are directed, the number of $\varepsilon$-tensors has to be even, in order to get a closed cycle:
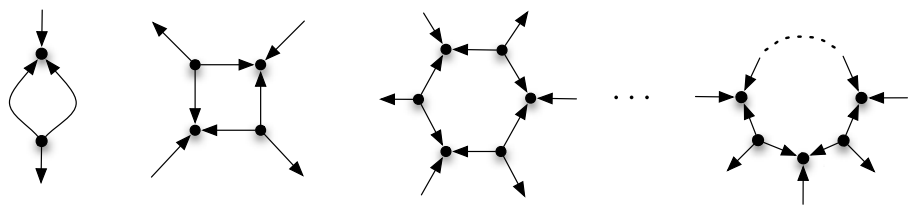

As a first instance, we will consider the meaning of a 2-cycle. This little gadget will turn out to be useful as an auxiliary calculation that will be needed in order to simplify the diagrams later on. The calculation is presented in the following diagram and explains how double arrows between $\varepsilon$-tensors can be replaced by a single arrow and a factor:

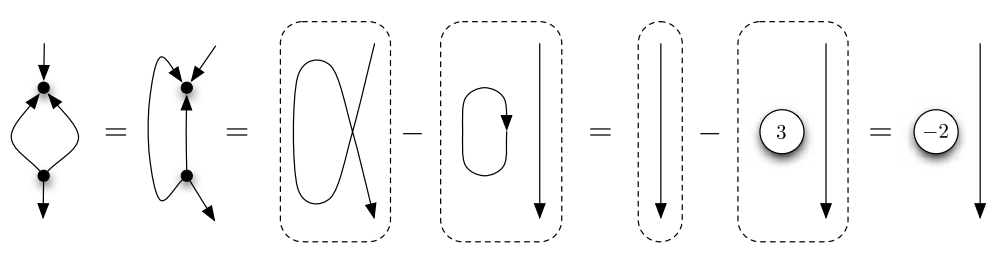

The first equality is just a topological deformation, the second is an application of the $\varepsilon$ - $\delta$-rule. The third equation is due to the fact that the closed loop $O=$ $\delta_{i}^{i}=3$ is nothing other than the trace of the unit matrix (here the dimension enters the calculation as a number).

Let us now consider larger cycles. Each of these cycles has an alternating sequence of ingoing and outgoing arrows along the boundary. We will analyze the cases in which we attach points and lines in an alternating manner. The vanishing of such a cycle encodes a projective condition that must be fulfilled by the points and lines that are involved. For each cycle, we will interpret this condition in two different ways. First we will give a geometric interpretation of such a cycle being closed for general cycles. After this we will analyze alternative geometric and algebraic characterizations by applying the $\varepsilon$ - $\delta$-rule.

Let us first consider a general $2 n$-cycle with points and lines attached alternatingly (as a running example, we consider a six-cycle. Our considerations, however, will apply for the general case). Assume that the points and lines are attached in the interleaved order $p_{1}, l_{1}, p_{2}, l_{2}, \ldots, p_{n}, l_{n}$. By breaking the cycle at a specific arrow between two $\varepsilon$-tensors (say the $\varepsilon$-tensor attached to $p_{1}$ and the $\varepsilon$-tensor attached to $l_{n}$ ) 
we derive a transformation $T$ that takes a point $q$ as input and "calculates" a point $q$ '.

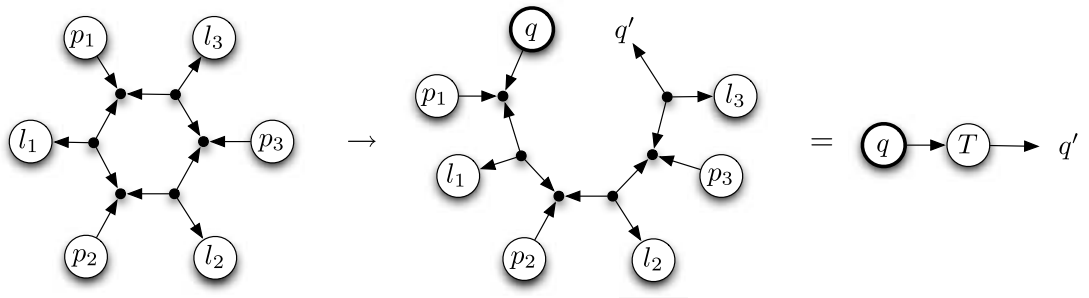

The vanishing of the closed cycle corresponds to the transformation $T$ having trace zero since $T$ by our cycle condition. Furthermore $T$, considered as a matrix, must have rank at most 2 since the transformed point $q^{\prime}$ will always lie on $l_{n}$. Geometrically this transformation corresponds to the a chain of join and meet operations, as indicated by the following picture:

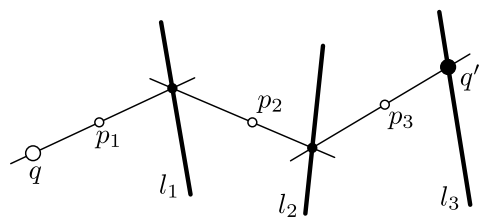

The final point $q^{\prime}$ will always lie on line $l_{n}$. Thus if we assume that point $q$ was chosen to lie also on $l_{n}$, then we obtain a (projective) transformation from $l_{n}$ to $l_{n}$. The cycle condition now translates to the following geometric criterion:

Theorem 3 With all settings as above we assume that the points and lines $p_{1}, l_{1}, p_{2}, l_{2}, \ldots, p_{n}, l_{n}$ are chosen such that the $\varepsilon$-cycle evaluates to zero. Then for an arbitrarily chosen line $g$, the following diagram will be zero as well:

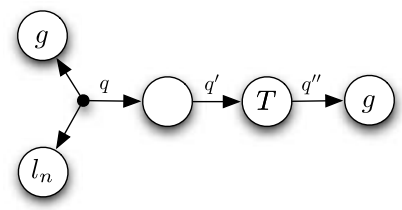

Before we prove this theorem we will investigate its geometric significance. If no degeneracy occurs along the construction, then intersecting $g$ with $l_{n}$ (the first $\varepsilon$-tensor), we get an arbitrary point $q$ on $l_{n}$. Applying the chain of joins and meets twice, we arrive at the point $q^{\prime \prime}$, which will again lie on $g$ and thus be identical to $q$ ( since it also lies on $l_{n}$ ). Thus either the sequence of operations degenerates at some point (which results in a zero tensor) or the mapping $T$ restricted to $l_{n}$ is an involution.

Proof of Theorem 3 We make simplifications according to the rule for trace-zero transformations derived at the beginning of Sect. 3.1 and to the $\varepsilon$ - $\delta$-rule (circles 
around tensors are omitted):

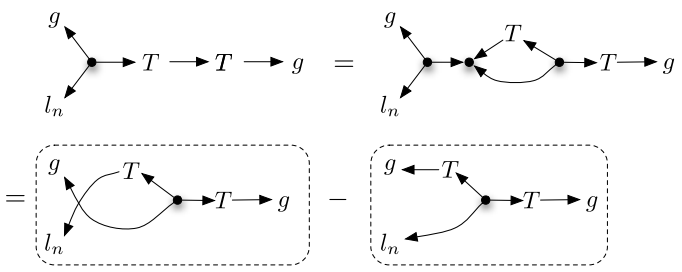

Both summands of the final expression vanish. The first one since $T$ produces a point that lies on $l_{n}$. The second one because of the fact that one $\varepsilon$-tensor is connected to two identical chains of tensors.

Now we will calculate algebraic expressions for the 4-cycle and for the 6-cycle. The 4-cycles turns out to be intimately related to harmonic relations of points. 6-cycles turn out to be related to Pappos's Theorem.

\subsubsection{4-Cycles}

A 4-cycle involves two points and two lines. If we apply the $\varepsilon$ - $\delta$-rule to a four cycle, we arrive at the following algebraic expression:

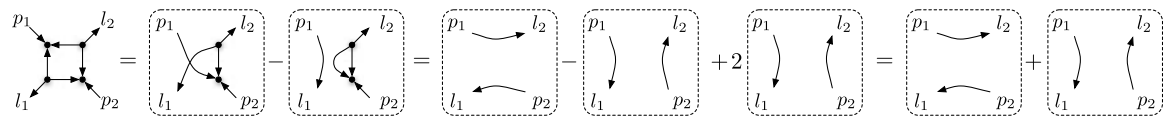

Vanishing of the final expression in vector language is $\frac{\left\langle p_{1}, l_{1}\right\rangle\left\langle p_{2}, l_{2}\right\rangle}{\left\langle p_{1}, l_{2}\right\rangle\left\langle p_{2}, l_{1}\right\rangle}=-1$. Which is the algebraic condition for the situation that the line joining $p_{1}$ and $p_{2}$ intersects $l_{1}$ and $l_{2}$ in two points that are harmonic to the pair $\left(p_{1}, p_{2}\right)$.

In Fig. 1 on the left a corresponding situation is shown in which $p_{1}, p_{2}, l_{1}, l_{2}$ satisfy the 4-cycle condition. The geometric statement derived by Theorem 3 reflects the algebraic condition for the harmonic situation. The algebraic reduction for the 4-cycle that we performed by the $\varepsilon-\delta$-rule is unique in the sense that, no matter in which order we perform $\varepsilon-\delta$-rules to the arrows, we will arrive at the same expression. Thus if we greedily perform $\varepsilon$ - $\delta$-rules whenever two $\varepsilon$-tensors are connected, we end up always with the same result.
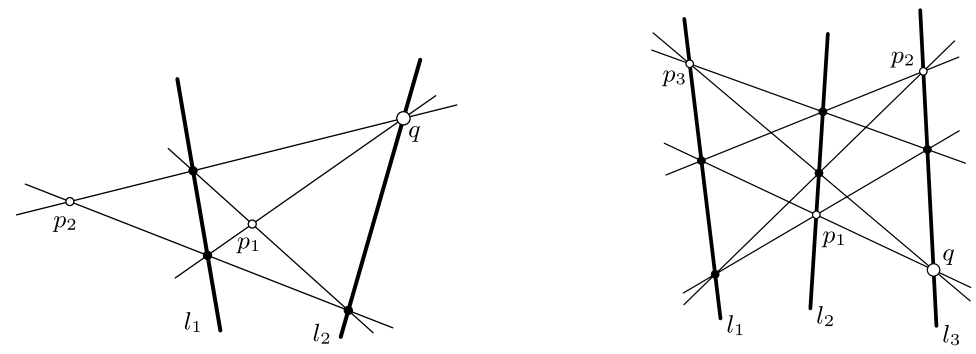

Fig. 1 Geometry of 4-cycles and 6-cycles 


\subsubsection{6-Cycles}

A similar calculation to the one above can be performed for 6-cycles. However, here the result of a greedy reduction process is not unique. We obtain (intermediate reduction steps omitted) the following two irreducible forms:
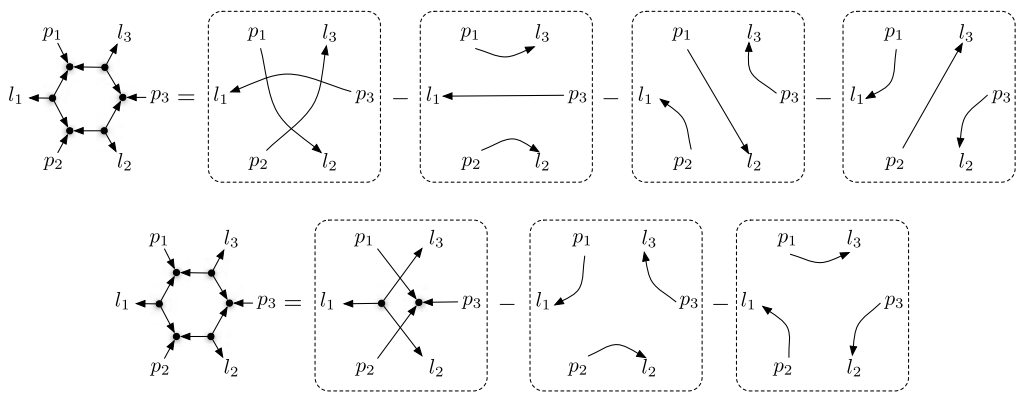

We now study particular geometric situations under which the 6-cycle expression vanishes. First of all, since the 6-cycle is linear in the points and lines, it follows that for every position of five of the elements, there is at least a one-dimensional space for the final object to obtain a vanishing cycle. We will now consider special cases in which the cycle vanishes. These come from the vanishing of the four summands in the first of the above expansions or from vanishing of the three summands in the second expansion. The summands consisting of three arrows will vanish if at least one of the point/line pairs connected by an arrow are incident. The summand with the two $\varepsilon$-tensors will vanish if either the three points are collinear or the three lines are concurrent. Whenever the cycle expression vanishes, Theorem 3 tells us that starting with a point $q$ on $l_{3}$ and performing a cycle of six consecutive join/meet operations (in the order specified by the cycle), we will either run in a degenerate situation, or the resulting point will coincide with $q$. A careful case by case analysis shows that most of these cases lead to degenerate situations. There is only one possibility of coincidences that makes the cycle expression vanish and does not lead to degeneracy. This is achieved if the pairs $\left(p_{1}, l_{2}\right),\left(p_{2}, l_{3}\right),\left(p_{3}, l_{1}\right)$ define coincidences. In this case the first expansion formula for the 6-cycle vanishes. The geometric situation is drawn in Fig. 1 on the right. If we start with a point $q$ on $l_{3}$ and cycle around twice (as indicated by Theorem 3), we must end up at the starting point $q$. This is nothing else than the well-known Pappos's Theorem.

Remark 1 Another interesting fact concerning the expansions of a 6-cycle arises if one considers the fact that both expansions must be algebraically the same. By this we obtain an algebraic expansion of the product of two determinants in terms of a linear combination of scalar products. Again removing the points and the lines from this expression, we get the following well-known identity on $\varepsilon$ and $\delta$ tensors:

$$
\varepsilon^{a b c} \varepsilon_{i j k}=\operatorname{det}\left(\begin{array}{ccc}
\delta_{i}^{a} & \delta_{i}^{b} & \delta_{i}^{c} \\
\delta_{j}^{a} & \delta_{j}^{b} & \delta_{j}^{c} \\
\delta_{k}^{a} & \delta_{k}^{b} & \delta_{k}^{c}
\end{array}\right)
$$

This is essentially equivalent to the expansion formula for determinants. 


\subsection{Invariants of Point and Line Configurations}

We already mentioned that every (relative) tensor invariant under linear transformations can be expressed as a linear combination of closed diagrams that only contain the tensors of the invariant, arrows, and co- or contravariant $\varepsilon$-tensors. (This is a translation of Theorem 17.2 in [18] on the level of diagrams.) Applying $\varepsilon-\delta$-rules until we get stuck, we can simplify such a closed diagram until we obtain a linear combination in which each summand is the product of factors of particularly simple types. In none of the factors two $\varepsilon$-tensors are connected. Thus each factor is either a point joined to a line (a scalar product), a contravariant $\varepsilon$-tensor attached to three points (a determinant of points) or a covariant $\varepsilon$-tensor attached to three lines (a determinant of lines).

We arrive at particularly interesting cases if either only lines or only points are involved. If only points are involved, then there cannot be a factor that includes any lines. Thus each summand of the invariant turns out to be the product of determinants. This is nothing but the well-known First Fundamental Theorem of Invariant Theory for vectors (points) under regular linear (projective) transformations. However it has been derived in a, say, very pedagogical and instructive way from a by far more general context.

A similarly interesting case arises if the original diagram depends on the same number of points as of lines. In this case by applying $\varepsilon$ - $\delta$-rules we end up with a linear combination, where in each summand the number of covariant and contravariant tensors must be the same. In this case we can use the formula derived in Remark 1 to replace the product of these $\varepsilon$-tensors by a linear combination of products of $\delta$ vectors and by this totally get rid of all $\varepsilon$-tensors. Thus in this case each diagram can be expressed as a linear combination of products of scalar products.

\subsection{Algebraic Curves}

So far the only geometric objects we studied were points, lines, and projective transformations. The diagrammatic method applies to a by far larger range of objects. Tensors with an arbitrary number of ingoing and outgoing arrows can be considered. Again the direction of the arrows determine whether the corresponding index should be transformed with $T$ or with $T^{-1}$. In particular symmetric tensors with only incoming arrows can be used to represent algebraic curves. Vanishing of closed diagrams again corresponds to projectively invariant properties. A wealth of interesting examples can be found, for instance, in [4-6]. Here we only review a few of the most basic facts.

Usually, a quadratic form on points in $\mathbb{R P}^{2}$ is given by a symmetric matrix $Q$. The points on the quadratic form are the nonzero solutions of the equation $p^{\mathrm{T}} Q p=0$. In tensor diagram notation the quadratic form corresponds to a symmetric tensor $Q^{i j}(\rightarrow Q \leftarrow)$, and the above expression becomes $P \rightarrow Q \hookrightarrow P$. Again the projectively invariant property that a point lies on a conic can be expressed as the vanishing of a closed diagram. Similarly a cubic can be expressed as a symmetric tensor $C^{i j k}$ with three ingoing arcs, and the corresponding condition that a point lies on the cubic is expressed by three copies of the point being pugged into these arrows. 
There are many surprisingly symmetric diagrams that express interesting geometric properties. Here we just mention the formulas (=diagrams) for a line $l$ being tangent to a conic $Q$ or a cubic $C$. A detailed derivation of these formulas in terms of tensor diagrams may be found in [6] or in [25].
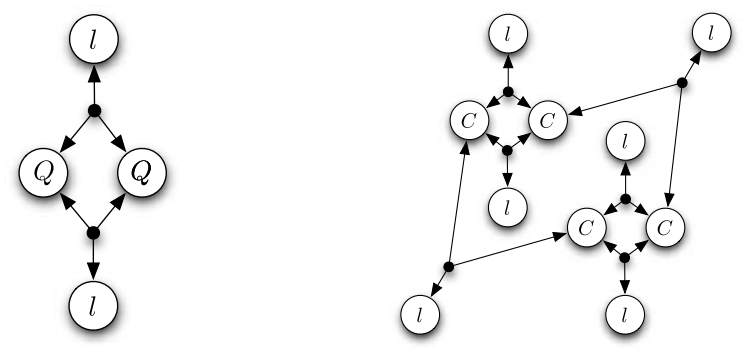

If we omit the two line tensors in the first diagram that corresponds to the conic, we are left with a diagram that transforms an arbitrary matrix $Q$ (also a nonsymmetric one) into its adjunct matrix (times a constant factor of -2 ). The second diagram is of degree six in the tensor $l$. It represents the (highly singular) sextic that encodes the polar of a cubic.

Although for algebraic curves still every (relative) invariant can be expressed as a linear combination of closed diagrams, it is by far more complicated to represent them in a standardized form as we did it in Sect. 3.3 for configurations of points and lines. The reason for this is that if a tensor has many connecting arrows, diagrams may occur that represent highly nontrivial graphs for which it is not possible to apply any $\varepsilon$ - $\delta$-rule. As an example of interesting small diagrams that represent invariants of curves, we consider the smallest nonzero irreducible diagrams that involve only one conic $Q$ or one cubic $C$ (perhaps many times).
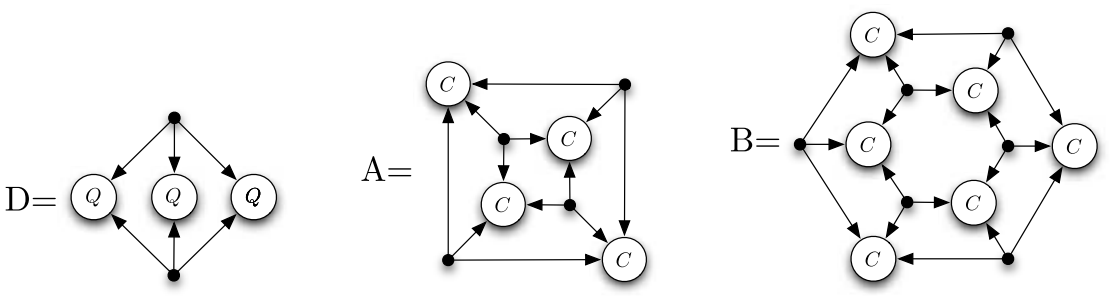

As mentioned in [6], the expressions $\Delta_{2}=-D / 6$ and $\Delta_{3}=(B / 6)^{2}-(A / 6)^{3}$ are the discriminants of the algebraic forms represented by $Q$ and $C$, respectively. In [23] the authors use diagram techniques to derive other invariants for algebraic forms in the context of curve recognition.

\subsection{Six Points on a Conic}

In this section we want to demonstrate how the $\varepsilon$ - $\delta$-rule helps to direct standard calculations into the "right" direction. We consider six points on a conic. By the wellknown Theorem of Pascal six points $a, b, c, d, e, f$ are on a conic if and only if in the 
incidence configuration shown in the picture below the points $x, y, z$ are collinear. The construction of the points $x, y, z$ can in a straightforward way be translated into a sequence of join and meet operations. Thus the collinearity of $x, y, z$ corresponds to the vanishing of a certain diagram, which turns out to be a tree of $\varepsilon$-tensors.
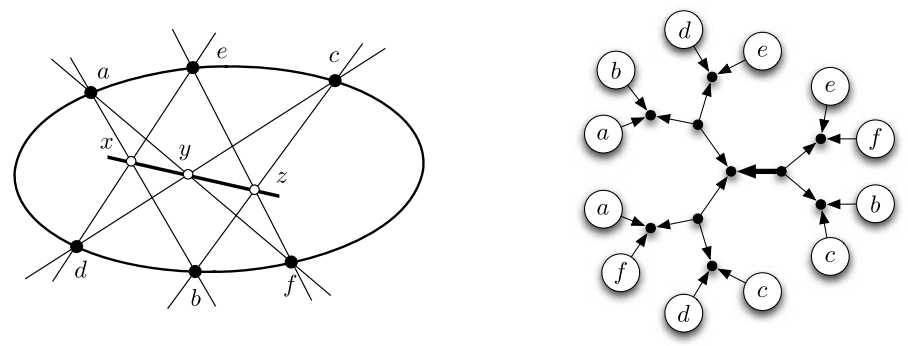

Applying the $\varepsilon$ - $\delta$-rule to the darkened edge, we arrive at the following diagram:
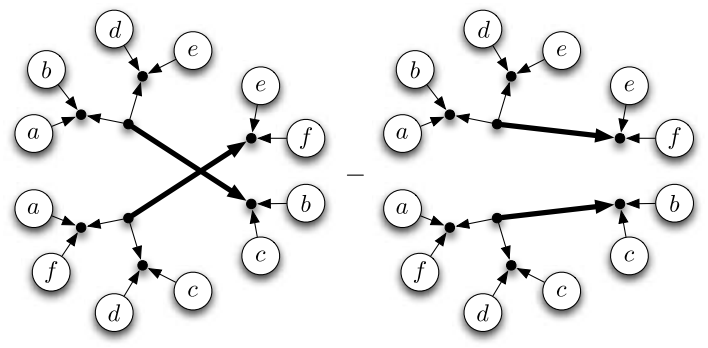

Applying the $\varepsilon$ - $\delta$-rule again to all connected sub-diagrams at the darkened edges, we have an intermediate expression in which every connected sub-diagram is just an $\varepsilon$ tensor with three soldered points. In half of these sub-diagrams one letter will appear twice (check it!). Collecting the remaining terms, we end up with

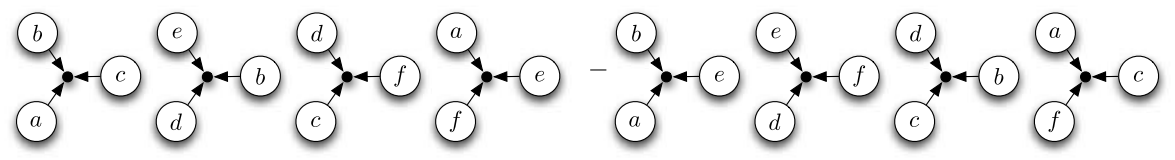

This is the well-known determinant condition for six points to lie on a conic. If we use $[p q r]$ as a shorthand for $\operatorname{det}(p, q, r)$, where $p, q, r$ are three-dimensional homogeneous coordinates for points in $\mathbb{R P}^{2}$, we arrive at

$$
[a b c][d e b][c d f][f a e]-[a b e][d e f][c d b][f a c]=0 .
$$

\section{Diagrams in Euclidean Geometry}

Diagrams can also be used to generate invariants for configurations under transformation groups other than the full projective transformation group. Following the spirit 
of Felix Klein's Erlanger Program and his approach to non-Euclidean geometry, the three types of geometries Euclidean, elliptic, and hyperbolic can be expressed by describing a fundamental object that remains invariant under the corresponding group of transformations. This fundamental object is a conic (or to be more precise, a pair of a primal and a dual quadratic form) that also may be degenerate. In the context of tensors we will represent these invariant objects again by suitably chosen tensors. We will now investigate how introducing these invariant objects into the diagram calculus immediately leads to geometrically meaningful diagrams.

\subsection{Euclidean Geometry}

We consider points represented by homogeneous coordinate vectors $(x, y, z)$. In the standard embedding on the $z=1$ plane Euclidean transformations are all those projective transformations that leave a certain pair of points $\{I, J\}$ invariant (as a pair). These two points have the complex coordinates $I=(i, 1,0)$ and $J=(-i, 1,0)$. Thus they both lie on the line at infinity, and both have complex coordinates. Rotations and translations leave the two points invariant individually, while reflections and glide reflections interchange them. As common in the theory, we will also consider similarities and similarities followed by (glide-)reflections as Euclidean transformations (they also leave the pair $\{I, J\}$ invariant). In the setup of Cayley-Klein geometry the pair $\{I, J\}$ plays the role of a dual degenerate conic (the fundamental object). The corresponding primal conic is the (doubly covered) line at infinity. All tangents to this fundamental object will pass through either of the points I or J. A euclidean (relative) invariant of a collection $X$ of geometric objects is a polynomial in the coordinate entries of $X$ such that $f(\tau(X))=\operatorname{det}(T)^{g} f(X)$ for every euclidean transformation $\tau$. Here $T$ is the corresponding $3 \times 3$ transformation matrix, and $g$ is a suitable integer.

If we want to represent euclidean invariants on the level of tensors (or tensor diagrams), we could draw diagrams involving the tensors of $X$ and I and J. Then we must take care of using $I$ and $J$ in a symmetric way in the diagram. However, we could also combine $I$ and $J$ right away in a symmetric and an antisymmetric manner and introduce two (covariant!) quadratic forms $E$ and $A$ (compare [31, 32]). In matrix/vector language the forms are defined as

$$
E=\left(\begin{array}{lll}
1 & 0 & 0 \\
0 & 1 & 0 \\
0 & 0 & 0
\end{array}\right)=\frac{\mathrm{IJ}^{\mathrm{T}}+\mathrm{JI}^{\mathrm{T}}}{2} \quad \text { and } \quad A=\left(\begin{array}{ccc}
0 & 1 & 0 \\
-1 & 0 & 0 \\
0 & 0 & 0
\end{array}\right)=\frac{I \mathrm{~J}^{\mathrm{T}}-\mathrm{JI}^{\mathrm{T}}}{2 i}
$$

Diagrammatically we get

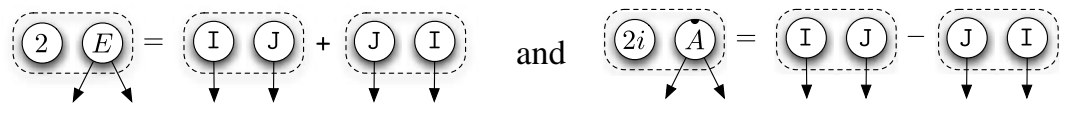

The tensors $E$ and $A$ are covariant (they have two outgoing arrows). Since I and J remain invariant (up to a factor) under rotations, the $E$ - and $A$-tensors are also invariant (up to a factor). While the $E$ tensor is symmetric, the $A$ tensor is antisymmetric. We indicate this in the diagrams by a little spot in the circle. Moving an arrow 
over another arrow or over the spot changes the sign. For rotations expressed by a projective $3 \times 3$ matrix $T$, we have

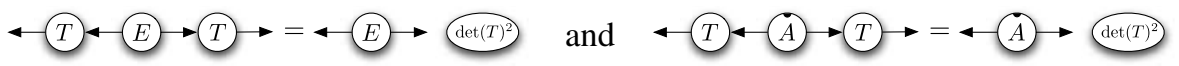

For orientation reversing euclidean transformations (they interchange $I$ and $\mathrm{J}$ ), the situation is slightly different. Here $E$ gets transformed as above. However, $A$ will change its sign.

The theory of euclidean invariants is essentially the theory of the tensors $\varepsilon, E$, and $A$. If $X$ are the tensors of geometric objects, then every closed diagram that involves these tensors and $\varepsilon, E$, and $A$ represents a euclidean invariant. Conversely, every euclidean invariant can be expressed as a linear combination of such diagrams. We will now first investigate the geometric meaning and interrelations of $E$ and $A$. After this we examine the geometric meaning of small diagrams and thereafter demonstrate how euclidean theorems can be proved by diagram techniques.

\subsection{The Tensors $A$ and $E$}

The tensor $A^{i j}$ can also be generated in a different way. Let $\infty^{i}=(0,0,1)$ be a tensor that represents the line at infinity. The line at infinity is spanned by $I$ and $J$ (in vector notation we have $\mathrm{I} \times \mathrm{J}=2 i \cdot \infty)$, and we have

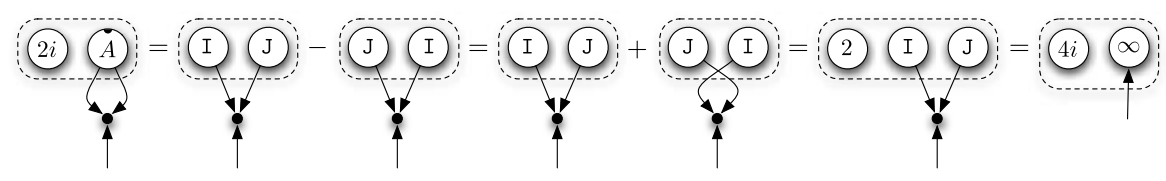

Thus $A_{i j} \varepsilon^{i j k}=2 \infty^{k}$. (This can also be proved by expanding the tensors.) Furthermore it is easy to prove that $\infty^{k} \varepsilon^{i j k}=-A_{i j}$, as the following diagram shows:

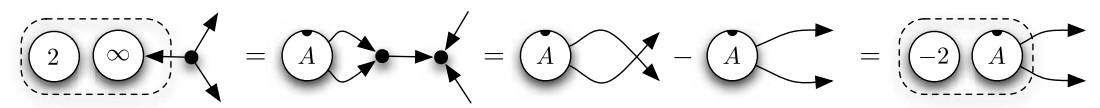

Thus in the presence of $\varepsilon$-tensors the $A$ tensor and the line at infinity are essentially the same. Diagrams that only involve the $A$ tensor, geometric objects and $\varepsilon$-tensors represent affine invariants.

In diagrams that contain both $A$ tensors and $E$ tensors we always may assume that there is at most one $A$ tensor involved. This is the case since we can replace any pair of $A$ tensors by a suitable linear combination of a pair of $E$ tensors, as the following equation shows:

$$
A_{i j} A_{k l}=E_{i l} E_{j k}-E_{i k} E_{j l} .
$$

This equation could again be easily proved either by expansion or by expanding the terms into linear combinations of I and J. Diagrams that only contain $E$ vectors are invariant under all euclidean transformations. Diagrams that contain an (odd number of) $A$ tensor(s) are only invariant under rotations (nevertheless setting such a diagram to zero may result in a euclidean invariant property). 


\subsection{Simple Diagrams and Their Meaning}

We now want to consider the simplest possible examples of diagrams that can be formed by $A, E, \varepsilon$-tensors and geometric objects and investigate their geometric significance. We assume that $p$ is a point, $l$ and $m$ are lines, and that $Q$ is a quadratic form. We will first consider the following list of diagrams:

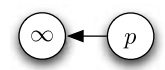

(a)

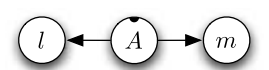

(b)

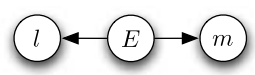

(c)

Diagram (a) being zero simply states that $p$ lies on the line at infinity. Diagram (b) being zero must encode an affinely invariant property. Expressing $A$ by $\infty^{k} \varepsilon^{i j k}$, we see that it encodes the fact that the meet of $l$ and $m$ is on the line at infinity. In other words, $l$ and $m$ are parallel. Diagram (c) is a bit less obvious. If vectorially $l=$ $\left(l_{1}, l_{2}, l_{3}\right)$ and $m=\left(m_{1}, m_{2}, m_{3}\right)$, then diagram (c) being zero encodes the algebraic condition $l_{1} m_{1}+l_{2} m_{2}=0$, which is nothing else than the condition that $l$ and $m$ are perpendicular. The ratio of the diagrams in (c) and (b) $\frac{(1)-(A)-(m)}{(1)-(B)}$ must be an absolute euclidean invariant, since it contains the vectors $l$ and $m$ in the same degree in numerator and denominator. In fact after a small and elementary calculation it turns out to be the arctan of the oriented enclosed angle between the two lines. In other words, one can encode the rotationally invariant condition that $l$ and $m$ enclose an angle of $\alpha$ by the vanishing of the diagram $\cos (\alpha) \cdot(b-A) \rightarrow-\sin (\alpha)$.

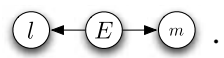

Let us now turn to quadratic forms. For this, let $Q$ be a contravariant quadratic form represented by a symmetric tensor. We investigate the following diagrams:

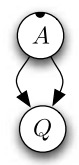

(d)

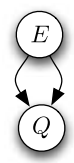

(e)

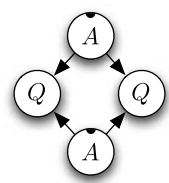

(f)

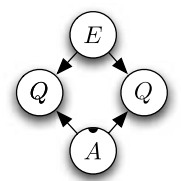

(g)

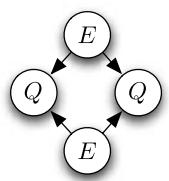

(h)

Let the algebraic form of the conic $Q$ be $a x^{2}+2 b x y+c y^{2}+($ lower order terms $)=0$. The coefficients $a, b, c$ are the only ones affected by the diagrams above. Since $Q$ is symmetric and $A$ is antisymmetric, the diagram $(d)$ must vanish identically for symmetry reasons. Diagram (e) is trace $\left(\begin{array}{ll}a & b \\ b & c\end{array}\right)=a+c$. Vanishing of $a+c$ encodes the geometric condition that the conic is a hyperbola with perpendicular asymptotes. Vanishing of diagram (f) can be nicely reduced to a condition at the line at infinity. If we replace $A$ by its representation by $\infty$ and $\varepsilon$, we get (up to an irrelevant factor):

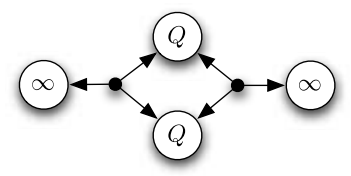


By our considerations of Sect. 3.4 this vanishing of this diagram geometrically expresses the fact that $Q$ is tangential to the line at infinity. In other words: $Q$ is $a$ parabola. In this case all tensors appear twice, and hence the sign of the diagram has also a geometric meaning. For a nonzero diagram, it distinguishes ellipses and hyperbolas. In order to get rid of the pair of $A$ tensors diagram (f) can also be expressed as diagram (h) minus the square of diagram (e). Diagram (g) vanishes identically for symmetry reasons. Diagram (h) is a bit subtle and requires a discussion of the underlying field. All involved matrices $E$ and $Q$ are $3 \times 3$. However, since in $E$ only the upper left $2 \times 2$ submatrix is nonzero, we may focus on this part of the tensors. On this part $E$ acts as a unit matrix, and the algebraic interpretation of this is $\operatorname{trace}\left(\left(\begin{array}{ll}a & b \\ b & c\end{array}\right)\left(\begin{array}{ll}a & b \\ b & c\end{array}\right)\right)=a^{2}+c^{2}+2 b^{2}$. If the tensors are supposed to be real numbers, this implies that the upper left $2 \times 2$ submatrix of $Q$ must be zero. Geometrically this means that $Q$ represents a degenerate conic that is composed of the line at infinity and an arbitrary other line.

There are also several interesting other invariants that can be expressed as linear combinations or rational functions of the diagrams (d) to (h). Consider, for instance, the difference of (h) and (f):

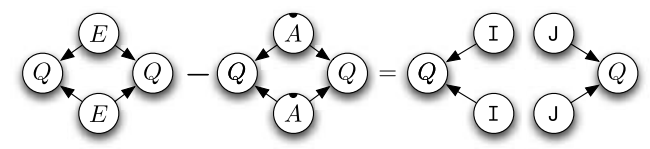

The equation follows from a straightforward calculation after expressing $E$ and $A$ in terms of I and J. Again we get an interesting interpretation over the reals. If the difference of (h) and (f) vanishes, then at least one of the two factors in the right part of the above equation will vanish. Say it is the first factor. This in turn means that $I$ lies on the conic $Q$. If the coefficient of the conic are real then also J must lie on $Q$. Both points I and J on $Q$ imply that $Q$ describes a circle.

\subsection{Four Points on a Circle}

How can we express diagrammatically that four points lie on a common circle? One would expect that this turns out to be a nicely symmetric property, and this is indeed the case. There are several ways to derive this condition. This time we will do it by translating a corresponding bracket expression from ordinary projective geometry to a tensor diagram setup. For this, let again $[p q r]$ be a shorthand for $\operatorname{det}(p, q, r)$. Every euclidean circle is a conic that passes through $I$ and $J$, and vice versa. Thus we can take the projective condition that six points are on a conic (see Sect. 3.5) and insert I and $\mathrm{J}$ as two of the points. In consequence four points $a, b, c, d$ lie on a conic if

$$
[a b \mathrm{I}][b c \mathrm{~J}][c d \mathrm{I}][d a \mathrm{~J}]-[a b \mathrm{~J}][b c \mathrm{I}][c d \mathrm{~J}][d a \mathrm{I}]=0 .
$$

(Compared to Sect. 3.5, the letters are slightly shuffled around according to the alternating determinant rules.) We will now try to express this condition by $A$ and $E$ tensors. As already mentioned before, $A$ is antisymmetric. Thus rotating it in a diagram by $180^{\circ}$ will change the sign of the diagram. Since $A$ and $E$ play a fundamental 
role anyway, we introduce new symbols for them, a symmetric one for $E$ and an antisymmetric one for $A$ :

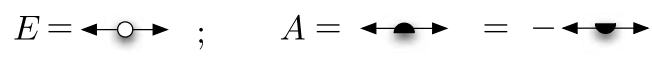

The relation between $A$ and $E$ tensors we encountered at the end of Sect. 4.2 can by this be written as

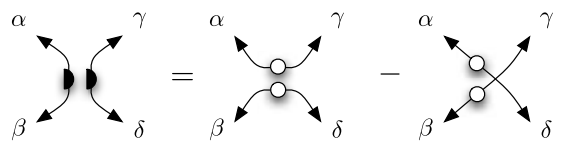

Transforming the index $\gamma$ by a matrix

$$
\left(\begin{array}{ccc}
0 & 1 & 0 \\
-1 & 0 & 0 \\
0 & 0 & 1
\end{array}\right)
$$

we get

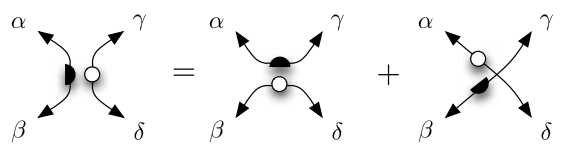

Before we come back to co-circular points, we derive another such relation that will turn out useful later on. For this, we rotate all diagrams in the above equation by $180^{\circ}$ and add the left and right sides to the equation above. We obtain

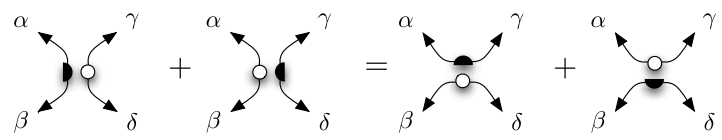

We now encode the bracket condition for co-circularity [abI] [bcJ][cdI][daJ]$[a b \mathrm{~J}][b c \mathrm{I}][c d \mathrm{~J}][d a \mathrm{I}]=0$ in terms of diagrams. Expressing the determinants by $\varepsilon$-tensors we get

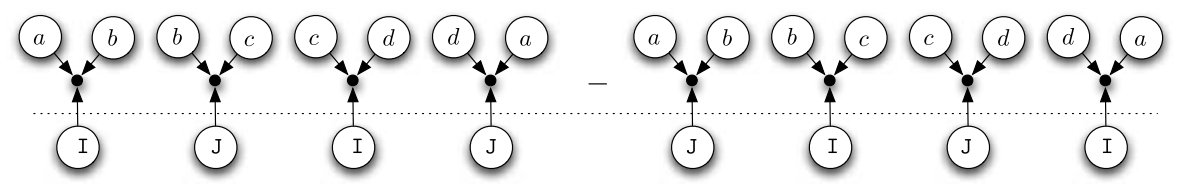

We inserted a dotted line to separate the structural part from the input data. A straightforward calculation going back to the definitions of $A$ and $E$ shows that we can replace the part under the dotted line (up to an irrelevant factor) in the following 
way:

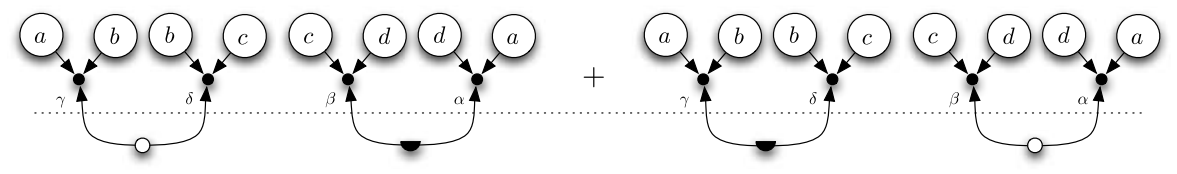

We omit the details of the calculation, but the reader should be aware of the following fact. If we replace the $A$ and $E$ tensors in the last diagram by their definition and multiply everything out, then both summands lead to altogether four terms. Collecting those terms, parts of both summands cancel out, and the remaining summands combine to the above bracket expression with I and $\mathrm{J}$.

Let us focus on what is above and below the dotted line. Above the dotted line, the $\varepsilon$-tensors essentially calculate the joins of consecutive pairs of points in the cyclic sequence $a, b, c, d$. Thus we may think of them as the sides $\gamma, \delta, \beta, \alpha$ of the quadrangle $a, b, c, d$. The co-circularity of $a, b, c, d$ entirely depends on the relative slopes of these lines. Below the dotted line, we get a nice symmetrization of $A$ and $E$. At first sight, it seems as if this formula singles out two opposite edges. However, using the formula on top of this page, we see that a cyclic shift of one index just reverses the sign of the formula. We may introduce a new diagram symbol for this special combination of $A$ and $E$ that graphically respects the symmetry of the combination:

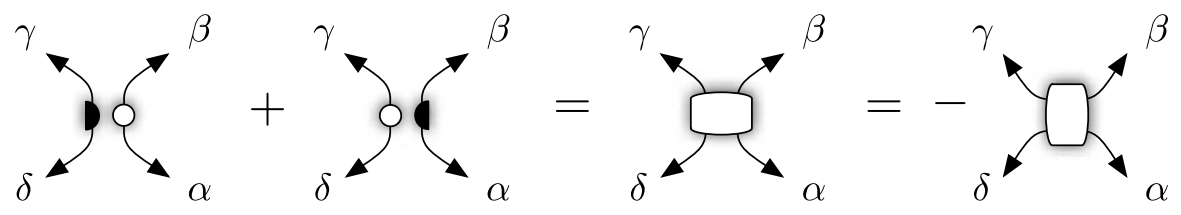

Rotating this symbol by $90^{\circ}$ reverses the sign. By this finally our diagram for co-circularity of four points becomes

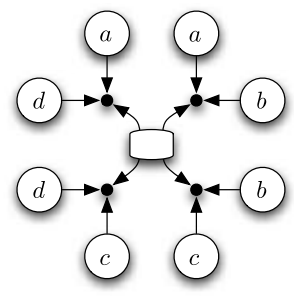

If this diagram vanishes, then the four points lie on a circle. Alternatively, one can also interpret this diagram differently. If three points are given, then we may interpret the diagram with two open arrows as a quadratic form describing a circle through these three points. 


\section{What We Did not Touch}

We just scratched the surface of the topic. A natural next step would be to exploit diagram techniques for sophisticated proving methods in geometry or for the derivation of invariants that are otherwise difficult to achieve. We want to end this paper with a few remarks on what happens when other transformation groups are involved.

\subsection{Spherical Transformations}

A very interesting topic arises when one studies spherical transformations. In spherical geometry the quadratic form associated to the fundamental object may be given as $x^{2}+y^{2}+z^{2}$. In tensor language it is represented by the covariant quadratic form that is given by the unit matrix. It may be represented by the symbol $\delta_{i j}$. We have seen in Sect. 3.4 that we may take $\varepsilon$-tensors to calculate the inverse of a quadratic form. Thus with $\delta_{i j}$ also the contravariant unit matrix $\delta^{i j}$ is present in our geometry. Both matrices stay invariant under spherical transformations.

We can use these matrices to convert any covariant index into a contravariant one. Thus we can turn any outgoing arrow into an ingoing one. In other words, the orientation is not relevant any more. Spherical geometry is the geometry of diagrams that only contain undirected edges and no arrows. One can also think about this a bit differently. The transformations of spherical geometry are exactly the $S O(n)$ matrices. For those matrices, we have $T=\left(T^{-1}\right)^{\mathrm{T}}$. Thus covariant and contravariant indices are transformed in the same way.

The symmetry in co- and contravariance has several consequences. Since we can replace any pair of co- and contravariant $\varepsilon$-tensors by a linear combination of arrows, we can (in spherical geometry) replace any pair of $\varepsilon$-tensors by a linear combination of edges. Thus any invariant in points and lines in spherical geometry is expressible as linear combination of diagrams that involve only scalar products and at most one determinant. In spherical geometry it is also reasonable to consider more sophisticated nets and cycles of $\varepsilon$-tensors, since there is no parity restriction. This leads to a whole new field of investigation.

\subsection{Diagrams in Quantum Information Theory}

One other group deserves considerable attention because of its relevance to physical applications: $S U(d)$. In quantum theory physical states of a quantum system are usually represented by tensors over the complex number field. Quantum systems evolve by hermitian operators on these tensors. In particular in quantum information theory one is interested in states of entangled qubits (like electron spin or photon polarization) or qutrits (like quark color). A single qubit (qutrit) is a vector of complex dimension 2 (dimension 3). A system of $n$ qubits or qutrits is represented by a vector whose array dimension is $n$. A state that corresponds to a classical (non-entangled) system is represented by a tensor that is simply the direct product of the vectors representing the classical qubits or qutrits. Entanglement is responsible for a certain nonlocal behavior (like the Einstein-Podolsky-Rosen paradox) that is characteristic for quantum theory. A system of $n$ such entangled particles may by this be described 
diagrammatically as a single node with $n$ outgoing arcs. A quantum measurement of a state is represented by a node with ingoing arcs. If a system with $n$ (possibly entangled) particles is distributed to $n$ observers, each of the observers (receivers of the outgoing arcs) can influence "its" particle by a local $S U(d)$ operation $(d=2$ for qubits and $d=3$ for qutrits).

An important question in quantum information theory is asking for a classification of so-called entanglement invariants. These are those numbers one can associate to a quantum system that do not depend on local $S U$ actions. These invariants again correspond to closed diagrams of a certain type. A little care is necessary here. As usual, the diagrams are composed from the physical state and $\varepsilon$-tensors. However, unlike in all the cases we considered so far, the outgoing edges are colored with respect to the individual particle they represent. Only edges of the same color may be connected (or connected to the same $\varepsilon$-tensor). Furthermore, according to the nature of the hermitian scalar product, the tensors involved in a diagram have to be complex conjugated and nonconjugated in alternating order. Hence the graph must be bipartite. The edges are as in the case of $S O$ undirected.

The geometric considerations of the previous sections give the possibility to interpret some of the quantum invariants on a geometric level. A further investigation in this direction is still work in progress. However, several surprising and interesting relations have already been discovered. For instance, in [29] a basis for a system of tensor invariants for a 3-qubit system is given. There are all together six such fundamental invariants given by the following diagrams:

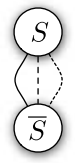

$I_{1}$

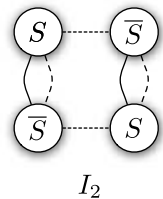

$I_{2}$

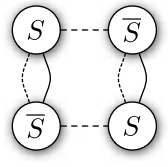

$I_{3}$

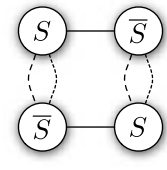

$I_{4}$

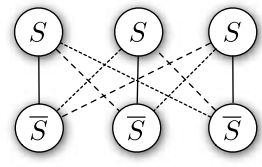

$I_{5}$

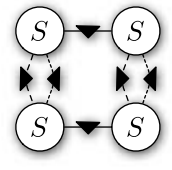

$I_{6}$

Here the coloring of the edges is represented by the different dashing type. The little triangle represents the matrix $\left(\begin{array}{cc}0 & 1 \\ -1 & 0\end{array}\right)$, which is the two-dimensional analogue of the $\varepsilon$-tensor. Surprisingly, some of these invariants have a geometrically meaningful interpretation. $I_{1}$ is just the squared norm of the state $S$. $I_{6}$ is Cayley's hyperdeterminant. Geometrically most interesting are $I_{2}, \ldots, I_{4}$. In a joint study [1] together with R. Bischoff the second author found the following geometric interpretation: The tensor representing the state $S$ is given by eight complex numbers. The shape of the tensor is best represented by a $2 \times 2 \times 2$ cube. We now split this cube into an upper and a lower half (each consisting of four coordinate entries) and consider these as vectors in $\mathbb{C}^{4}$. Taking the exterior product of these two vectors produces a six-dimensional vector which is up to a scalar multiple invariant under two of the $S U(2)$ actions under consideration. Taking the norm of this vector produces a number which is also invariant to the third $S U(2)$ action (as a consequence of the Cauchy-Binet theorem). This invariant turns out to be a linear combination of the squared norm $I_{1}$ and one of the invariants $I_{2}, \ldots, I_{4}$. The other invariants become involved by splitting the cube in a different direction. The proof of this fact can be nicely carried out on the level of diagrams. In fact, these diagrams somehow also involve translation tensors from 
$\mathbb{C}^{2}$ to $\mathbb{C}^{4}$. The Cauchy-Binet formula turns out to be a special case of the $\varepsilon$ - $\delta$-rule. It seems to be a promising direction to interpret concepts from quantum information theory in terms of projective geometry. Tensor diagrams appear to be a very useful tool in this context.

Acknowledgement The authors want to thank Jim Blinn for interesting and helpful comments and remarks that definitely improved our expositions.

\section{References}

1. Bischoff, R.: Geometry of qubits. Diploma thesis, ETH Zurich (2003)

2. Blinn, J.F.: Uppers and downers, part 1. IEEE Comput. Graph. Appl. 12(2), 85-91 (1992)

3. Blinn, J.F.: Uppers and downers, part 2. IEEE Comput. Graph. Appl. 12(3), 80-85 (1992)

4. Blinn, J.F.: Polynomial discriminants, part 1: matrix magic. IEEE Comput. Graph. Appl. 20(6), 94-98 (2000)

5. Blinn, J.F.: Polynomial discriminants, part 2: tensor diagrams. IEEE Comput. Graph. Appl. 21(1), 86-92 (2001)

6. Blinn, J.F.: Quartic discriminants and tensor invariants. IEEE Comput. Graph. Appl. 22(2), 86-91 (2002)

7. Blinn, J.F.: Lines in space, part 1: the 4D cross product. IEEE Comput. Graph. Appl. 23(2), 84-91 (2003)

8. Blinn, J.F.: Lines in space, part 2: the line formulation. IEEE Comput. Graph. Appl. 23(3), 72-79 (2003)

9. Blinn, J.F.: Lines in space, part 3: the two matrices. IEEE Comput. Graph. Appl. 23(4), 96-101 (2003)

10. Blinn, J.F.: Lines in space, part 4: back to the diagrams. IEEE Comput. Graph. Appl. 23(5), 84-93 (2003)

11. Blinn, J.F.: Lines in space, part 5: a tale of two lines. IEEE Comput. Graph. Appl. 23(6), 84-97 (2003)

12. Blinn, J.F.: Lines in space, part 6: our friend the hyperbolic paraboloid. IEEE Comput. Graph. Appl. 24(3), 92-100 (2004)

13. Blinn, J.F.: Lines in space, part 7: the algebra of tinkertoys. IEEE Comput. Graph. Appl. 24(4), 96-102 (2004)

14. Blinn, J.F.: Lines in space, part 8: line(s) through four lines. IEEE Comput. Graph. Appl. 24(5), 100106 (2004)

15. Clifford, W.: Extract of a letter to Mr. Sylvester from Prof. Clifford of University College. Am. J. Math. 1, 126-128 (1878)

16. Cvitanović, P.: Group Theory: Birdtracks, Lie's, and Exceptional Groups. Princeton University Press, Princeton (2008)

17. Dolotin, V., Morozov, A.: Introduction to Non-Linear Algebra. World Scientific, Singapore (2007)

18. Gurevich, G.B.: Foundations of the Theory of Algebraic Invariants. Noordhoff, Groningen (1964)

19. Kempe, A.B.: On the application of the Sylvester-Clifford graphs to ordinary binary quantics. Proc. Lond. Math. Soc. 17, 107-121 (1885)

20. Kortenkamp, U., Richter-Gebert, J.: Grundlagen dynamischer Geometrie, Zeichnung-Figur-Zugfigur, 123-144 (2001)

21. Lebmeir, P., Richter-Gebert, J.: Recognition of computationally constructed loci. In: Lecture Notes in Computer Science/Artificial Intelligence, vol. 4869, pp. 52-68. Springer, Berlin (2007)

22. Lebmeir, P., Richter-Gebert, J.: Rotations, translations and symmetry detection for complexified curves. Comput. Aided Geom. Design (Special issue on Classical Techniques for Applied Geometry) 25, 707-719 (2008)

23. Lebmeir, P., Feature detection for real plane algebraic curves. Doctoral thesis, Technical University Munich (2009)

24. Olver, P.J.: Classical Invariant Theory. Cambridge University Press, Cambridge (1999)

25. Richter-Gebert, J.: Hands on Projective Geometry, book in preparation. See http://www-m10.ma.tum. de/twiki/bin/view/Lehre/ProjektiveGeometrieWS0607

26. Richter-Gebert, J., Kortenkamp, U.: Cinderella-The Interactive Geometry Software. Springer, Berlin (1999) (see also http://www.cinderella.de) 
27. Stedman, G.E.: Diagram Techniques in Group Theory. Cambridge University Press, New York (1990)

28. Sturmfels, B.: Algorithms in Invariant Theory. Springer, Wien/New York (1993)

29. Sudbery, A.: On local invariants of three-qubit states. J. Phys. A 34, 643-652 (2000) (quant-ph/ 0001116)

30. Sylvester, J.: On an application of the new atomic theory to the graphical representation of the invariants and covariants of binary quantics, with three appendices. Am. J. Math. 1, 64-125 (1878)

31. Wade, T.L.: Euclidean concommitants of the tenary cubic. Bull. Am. Math. Soc. 48(8), 589-601 (1942)

32. Wade, T.L.: Euclidean concommitants of the triangle. Natl. Math. Mag. 17(8), 335-340 (1943)

33. Wade, T.L.: Tensor algebra and invariants, part I. Natl. Math. Mag. 19(1), 3-10 (1944)

34. Wade, T.L.: Tensor algebra and invariants, part II. Natl. Math. Mag. 20(1), 5-10 (1945)

35. Weyl, H.: The Classical Groups. Princeton University Press, Princeton, New Jersey (1939) 